\title{
Les gens qui fument prennent des pauses!
}

\section{HRONIQUE • Insertion professionnelle}

Loin de moi l'idée de faire de ce texte une incitation à fumer, mais plutôt à prendre des pauses dans le cadre du travail! J'évoque en fait cette image parce que l'idée de cette chronique m'est venue alors que j'étais en train de diner devant mon ordinateur quand j'ai vu une collègue passer pour aller fumer... On ne se permet pas toujours de prendre une pause, voire de s'arrêter pour diner (!), mais le besoin de griller une cigarette incite, lui, à prendre régulièrement quelques minutes pour s'arrêter et aller prendre l'air.

Surtout en tant qu'enseignant débutant, en début d'année scolaire ou en période de bulletins de surcroît, on oublie trop souvent que les pauses sont nécessaires. Voici donc quelques pistes pour nous rappeler leur importance et réussir à les intégrer au quotidien.

\section{Assainir sa relation au travail en cherchant un équili- bre entre travail et vie personnelle}

Un certain nombre de chercheurs qui se sont intéressés à la résilience des enseignants, surtout les débutants, ont mis en évidence divers facteurs de protection individuels qui réfèrent à l'idée de prendre soin de soi. Parmi ceux-ci, la recherche d'un certain équilibre entre le travail et la vie personnelle occupe une place de choix (Castro, Kelly et Shih, 2010). Cela renvoie aux limites que l'on se donne, à la coupure qu'on fait entre le temps professionnel et le temps personnel. Une enseignante rencontrée dans le cadre de ma recherche doctorale avait proposé une image 


\section{CHROMLOLIIE}

intéressante en ce sens en affirmant : «Le soir, je n'apporte pas mon sac d'école à la maison, comme ça, je ne traîne pas de coquerelles! ». Cette coupure peut se faire en s'assurant de préserver du temps pour sa famille et ses amis ou pour faire de l'exercice ou relaxer (Sharplin, O’Neill et Chapman, 2011), en s'astreignant à quitter physiquement le lieu du travail (Castro et al., 2010).

\section{Viser la santé psychologique et éviter l'épuisement}

Plusieurs études se sont aussi intéressées aux liens à établir entre la santé physique et psychologique et la performance au travail (Ford, Cerasoli, Higgins et Decesare, 2011). Ces études permettent de mieux comprendre que « l'amélioration de la santé psychologique globale et de la satisfaction avec sa vie vont de pair avec une meilleure performance au travail, de même qu'avec un faible niveau de dépression et d'anxiété » (Théorêt et Leroux, 2014, p. 70).

Parallèlement, bon nombre d'études portant sur l'épuisement professionnel révèlent que la fatigue émotionnelle est un des principaux éléments nocifs à contrer. En ce sens, le détachement psychologique et l'imposition de frontières entre la maison et l'école seraient favorables au bien-être de l'enseignant (Théorêt et Leroux, 2014). Il conviendrait donc de s'obliger à prendre des pauses et à se fixer des limites dans le cadre du travail. Par exemple : profiter de la récréation pour aller faire une courte promenade autour de l'école ou aller discuter de sujets informels à la salle des enseignants, prendre du temps pour se faire un thé et lire ou aller courir à l'heure du dîner, prendre un moment calme et fermer les yeux une fois les élèves partis, mettre une minuterie (p. ex., 45-60 minutes) pour faire de la correction ou de la planification après l'école puis partir ensuite, etc. On peut même dresser une liste d'activités plaisantes pour soi, de durée courte ou indéterminée, dans laquelle on puisera le moment de la pause venu (Théorêt et Leroux, 2014), peu importe qu'on y consacre 5 ou 30 minutes.

Bref, prendre le temps de s'occuper de sa propre santé ne serait pas une perte de temps comme on a tendance à le croire (on s'en culpabilise souvent même), mais il s'agirait plutôt d'une forme d'investissement à court, moyen ou long terme! L'idée est de ne pas entretenir l'ILLUSION que les pauses servent à prendre de l'avance dans son travail, mais plutôt à en sortir et à décrocher pour pouvoir y revenir en meilleure disposition (Théorêt et Leroux, 2014).

\section{Intégrer la relaxation au travail}

Certes, des moments de relaxation peuvent être intégrés à notre quotidien de diverses manières. Or, depuis quelques années, un fort mouvement autour de la présence attentive ou de la pleine conscience (mindfulness) se fait sentir. L'idée est en quelque sorte de freiner la course folle que nous entretenons, cette fuite en avant vers un idéal toujours croissant de performance et de productivité, puis de prendre un temps d'arrêt pour se recentrer sur l'instant présent. On peut par exemple fermer les yeux et essayer d'observer attentivement ce que l'on ressent pour décrire finement les diverses stimulations internes et externes vécues dans le moment présent (Théorêt et Leroux, 2014). Ce type d'exercice est de plus en plus mis en œuvre dans divers contextes, dont celui des enseignants (Jennings, Snowberg, Coccia et Greenberg, 2011; Roeser, Skinner, Beers et Jennings, 2012). Et on peut même le faire avec les élèves afin de les amener eux aussi à intégrer cette habitude à leur quotidien! Malboeuf-Hurtubise et Lacourse (2016) suggèrent d'ailleurs plusieurs exercices pertinents en ce sens. 
Sur ce, je vais m'arrêter et prendre quelques instants pour relire moi-même cette chronique et tenter encore une fois de la mettre en pratique! Rappelons-nous que changer ses habitudes exige du temps et de la persévérance, car une nouvelle habitude se construit dans la durée et la répétition (Théorêt et Leroux, 2014). Et pourquoi ne pas impliquer aussi quelques amis et collègues dans cette démarche? C'est toujours plus motivant de pouvoir s'encourager mutuellement!

\section{Références}

Castro, A. J., Kelly, J. et Shih, M. (2010). Resilience strategies for new teachers in high-needs areas. Teaching and Teacher Education, 26(3), 622-629. http://dx.doi.org/10.1016/j.tate.2009.09.010

Ford, M. T., Cerasoli, C. P., Higgins, J. A. et Decesare, A. L. (2011). Relationships between psychological, physical, and behavioural health and work performance: A review and meta-analysis. Work E' Stress, 25(3), 185-204. http://dx.doi.org/10.1080/02678373.2011.609035

Jennings, P. A., Snowberg, K. E., Coccia, M. A. et Greenberg, M. T. (2011). Improving classroom learning environments by cultivating awareness and resilience in education (CARE): results of two pilot studies. Journal of Classroom interaction, 46(1), 37-48. Repéré à

http://prevention.psu.edu/projects/documents/Jennings CARE JClassInteract 2011.pdf

Malboeuf-Hurtubise, C. et Lacourse, E. (2016). Mission Méditation : pour des élèves épanouis, concentrés et ouverts. Québec, QC : Éditions Midi Trente.

Roeser, R. W., Skinner, E., Beers, J. et Jennings, P. A. (2012). Mindfulness training and teachers' professional development: An emerging area of research and practice. Child Development Perspectives, 6(2), 167-173. http://dx.doi.org/10.1111/j.1750-8606.2012.00238.x

Sharplin, E., O’Neill, M. et Chapman, A. (2011). Coping strategies for adaptation to new teacher appointments: Intervention. Teaching and Teacher Education, 27(1), 136-146. http://dx.doi.org/10.1016/j.tate.2010.07.010

Théorêt, M. et Leroux, M. (2014). Comment améliorer le bien-être et la santé des enseignants? Des ressources pour la résilience éducationnelle. Louvain-la-Neuve : De Boeck Supérieur.

\section{Pour citer cet article}

Leroux, M. (2016). Les gens qui fument prennent des pauses! Formation et profession. 24(3), 87-89. http://dx.doi.org/10.18162/fp.2016.a105 